\title{
Molecule 4
}

\section{Singlet}

\section{UHF/STO-3G}

1 | 1 | UNPC-UNK |FOpt | UHF |STO-3G |C10H8N2 | PCUSER | 19-Oct-2002|0||\#UHF STO-3G OPT GUESS $=($ ALWAYS, MIX) || molecule 54||0,1|C,1.5367021694,0.,0.55092701 $48|\mathrm{C}, 1.551572589,0 ., 1.967375894| \mathrm{H}, 2.5105424165,0 ., 2.472219157 \mid \mathrm{C}, 0.3541$ $875581,0 ., 2.7470978241|\mathrm{H}, 0.4293220871,0 ., 3.8270613411| \mathrm{C},-0.8673716891$, $0 ., 2.1317872981|\mathrm{H},-1.7890585062,0 ., 2.6993257403| \mathrm{C},-0.969249135,0 ., 0.63$ $06098482|\mathrm{C},-2.2686108602,0 ., 0.0403412368| \mathrm{H},-3.1393089259,0 ., 0.68337855$ $22|\mathrm{C},-2.419009737,0 .,-1.3554970571| \mathrm{H},-3.4055345745,0 .,-1.8010691069 \mid \mathrm{C}$, $-1.2744707133,0 .,-2.1691358554|\mathrm{H},-1.3750573685,0 .,-3.2479175888| \mathrm{C}, 0.04$ $41170949,0 .,-1.6056206039|\mathrm{~N}, 1.1829013483,0 .,-2.4408814306| \mathrm{H}, 0.79239667$ $24,0 .,-3.4216141451|\mathrm{C}, 0.1825586838,0 .,-0.1557116198| \mathrm{N}, 2.7125513945,0 .$, $-0.1919134203|\mathrm{H}, 3.4859732363,0 ., 0.5251361269|$ |Version=x86-Win32-G98Rev A.11.4 State $=1-A^{\prime}|H F=-486.1370331| S 2=2.403761|S 2-1=0| S 2 A=8.901585 \mid$. RMS $\mathrm{D}=8.774 \mathrm{e}-009|\mathrm{RMSF}=4.144 \mathrm{e}-005| \mathrm{Dipole}=-0.8835799,0 ., 0.6199271 \mid \mathrm{PG}=\mathrm{CS} \quad[\mathrm{SG}($ $\mathrm{C} 10 \mathrm{H} 8 \mathrm{~N} 2)]|| \mathrm{e}$

\section{UCCSD(T)/STO-3G}

$1|1|$ UNPC-UNK $\mid$ SP | UCCSD (T) -FC |STO-3G|C10H8N2 | PCUSER | 19-OCt-2002|0|| \#UCCS D-T/STO-3G GUESS = (ALWAYS, MIX) || molecule 54||0,1|C|C, 1, 1.41652693|H, 2, 1 $.08373883,1,118.3657532|\mathrm{C}, 2,1.4288797,1,122.47017331,3,180 ., 0| \mathrm{H}, 4,1.08$ $257397,2,119.0919315,1,180 ., 0|\mathrm{C}, 4,1.36777704,2,120.19352381,1,0 ., 0| \mathrm{H}, 6$ $, 1.08240772,4,121.64206252,2,180 ., 0 \mid C, 6,1.50463044,4,120.61723853,2,0$. $, 0|\mathrm{C}, 8,1.42715028,6,118.31357631,4,180 ., 0| \mathrm{H}, 9,1.08241032,8,119.1218414$ $7,6,0 ., 0|\mathrm{C}, 9,1.40391751,8,120.58093359,6,180 ., 0| \mathrm{H}, 11,1.08248127,9,120$. $45648946,8,180 ., 0|\mathrm{C}, 11,1.40427122,9,119.25877195,8,0 ., 0| \mathrm{H}, 13,1.0834609$ $8,11,120.08164318,9,180 ., 0|\mathrm{C}, 13,1.43395371,11,121.45138776,9,0 ., 0| \mathrm{N}, 15$ $, 1.41226422,13,120.60112064,11,180 ., 0 \mid \mathrm{H}, 16,1.05561857,15,104.5475401,1$ $3,0 ., 0|\mathrm{C}, 15,1.45650339,13,118.59429137,11,0 ., 0| \mathrm{N}, 1,1.39083907,18,120.1$ $6032943,15,0 ., 0|\mathrm{H}, 19,1.05467597,1,104.88342728,18,180 ., 0| \mid$ Version=x86Win32-G98RevA.11.4 |State $=1-A^{\prime}|\mathrm{HF}=-486.1370331|$ MP $2=-486.6731755 \mid$ MP $3=-48$ $6.7686202|\mathrm{MP} 4 \mathrm{D}=-486.8006692| \mathrm{MP} 4 \mathrm{DQ}=-486.7941618|\mathrm{PUHF}=-485.5235366| \mathrm{PMP} 2-$ $0=-486.0544971|\mathrm{PMP} 3-0=-486.1414355| \mathrm{MP} 4 \mathrm{SDQ}=-486.8090535 \mid \mathrm{CCSD}=-486.88309$ $63|\operatorname{CCSD}(\mathrm{T})=-486.9031148| \mathrm{S} 2=2.403761|\mathrm{~S} 2-1=2.079876| \mathrm{S} 2 \mathrm{~A}=8.901585 \mid \mathrm{RMSD}=8$. $774 \mathrm{e}-009|\mathrm{PG}=\mathrm{CS} \quad[\mathrm{SG}(\mathrm{C} 10 \mathrm{H} 8 \mathrm{~N} 2)]| \mid \mathrm{e}$

\section{UB3LYP/STO-3G}

1 | 1 | UNPC-UNK | SP | UB3LYP |STO-3G |C10H8N2 |PCUSER $02-F e b-2003|0| \mid$ \#UB3LYP / ST O-3G GUESS $=($ ALWAYS, MIX) || molecule 54||0,1|C|C, 1, 1.41652693|H, 2, 1.08373 $883,1,118.3657532|\mathrm{C}, 2,1.4288797,1,122.47017331,3,180 ., 0| \mathrm{H}, 4,1.08257397$ , 2,119.0919315,1,180.,0|C,4,1.36777704,2,120.19352381,1,0.,0|H,6, 1.082 $40772,4,121.64206252,2,180 ., 0|C, 6,1.50463044,4,120.61723853,2,0 ., 0| C, 8$ $, 1.42715028,6,118.31357631,4,180 ., 0 \mid \mathrm{H}, 9,1.08241032,8,119.12184147,6,0$. $, 0|\mathrm{C}, 9,1.40391751,8,120.58093359,6,180 ., 0| \mathrm{H}, 11,1.08248127,9,120.456489$ $46,8,180 ., 0|\mathrm{C}, 11,1.40427122,9,119.25877195,8,0 ., 0| \mathrm{H}, 13,1.08346098,11,1$ 
$20.08164318,9,180,0|\mathrm{C}, 13,1.43395371,11,121.45138776,9,0 ., 0| \mathrm{N}, 15,1.412$ $26422,13,120.60112064,11,180 ., 0 \mid \mathrm{H}, 16,1.05561857,15,104.5475401,13,0 ., 0$ $|\mathrm{C}, 15,1.45650339,13,118.59429137,11,0 ., 0| \mathrm{N}, 1,1.39083907,18,120.1603294$ $3,15,0 ., 0|\mathrm{H}, 19,1.05467597,1,104.88342728,18,180 ., 0| \mid$ Version=x86-Win32G98RevA.11.4 State=1-A' $|\mathrm{HF}=-489.1017513| \mathrm{S} 2=1.068548|\mathrm{~S} 2-1=0.| \mathrm{S} 2 \mathrm{~A}=0.5981$ $84|\mathrm{RMSD}=7.095 \mathrm{e}-005| \mathrm{Dipole}=-0.9683275,0 ., 0.6497581|\mathrm{PG}=\mathrm{CS} \quad[\mathrm{SG}(\mathrm{C} 10 \mathrm{H} 8 \mathrm{~N} 2)]|$ | @

\section{UHF/4-31G}

1 | 1 | UNPC-UNK |FOpt | UHF | 4-31G | C10H8N2 | PCUSER | 05-DeC-2002|0|| \#UHF 4-31G 0 PT GUESS $=($ ALWAYS, MIX) || molecule 54 || $0,1 \mid C, 1.5058690101,0 ., 0.5560433077$ |C,1.499765033,0.,1.9638218786| H, 2.4463242944,0.,2.4699994847|C, 0.3218 $537104,0 ., 2.7106675013|\mathrm{H}, 0.3642146362,0 ., 3.7812070677| \mathrm{C},-0.8778105994$, $0 ., 2.0578853528|\mathrm{H},-1.8011409718,0 ., 2.6019155271| \mathrm{C},-0.9302644329,0 ., 0.6$ $187600593|\mathrm{C},-2.2372834813,0 ., 0.0141051863| \mathrm{H},-3.0958661691,0 ., 0.6554706$ $021|\mathrm{C},-2.3756313426,0 .,-1.344545858| \mathrm{H},-3.3464939278,0 .,-1.7976086423 \mid \mathrm{C}$ $,-1.231413081,0 .,-2.1422627767|\mathrm{H},-1.3325372544,0 .,-3.210892193| \mathrm{C}, 0.069$ $203209,0 .,-1.6037916296|\mathrm{~N}, 1.1381960497,0 .,-2.4211212892| \mathrm{H}, 0.8597987325$ $, 0 .,-3.3928680952|\mathrm{C}, 0.2168179206,0 .,-0.1441983038| \mathrm{N}, 2.6728845009,0 .,-0$ $.1138308387|\mathrm{H}, 3.4615011284,0 ., 0.5185328372| \mid$ Version=x86-Win32-G98RevA. $11.4 \mid$ State=1-A' $|\mathrm{HF}=-491.4677471| \mathrm{S} 2=1.623602|\mathrm{~S} 2-1=0.| \mathrm{S} 2 \mathrm{~A}=4.714562 \mid \mathrm{RMSD}=$ $5.799 e-009|\operatorname{RMSF}=1.333 e-004|$ Dipole $=-1.1957887,0 ., 0.7953091 \mid \mathrm{PG}=\mathrm{CS} \quad[\mathrm{SG}(\mathrm{C} 1$ $0 \mathrm{H} 8 \mathrm{~N} 2)]|| \mathrm{e}$

\section{UCCSD(T)/4-31G}

1 | 1 | UNPC-UNK |SP | UCCSD (T) -RW | 4-31G |C10H8N2 |PCUSER | 25-Mar-2003|0 || \#UCCSD $(\mathrm{T}, \mathrm{WINDOW}=(13,110)) / 4-31 \mathrm{G}$ GUESS $=($ ALWAYS, MIX $)||$ molecule 54|| $0,1|\mathrm{C}| \mathrm{C}, 1,1$ $.4077918|\mathrm{H}, 2,1.07340123,1,117.88745411| \mathrm{C}, 2,1.39472344,1,122.62485351,3$ $, 180 ., 0|\mathrm{H}, 4,1.07137734,2,120.11043227,1,180 ., 0| \mathrm{C}, 4,1.36576681,2,119.07$ $131078,1,0 ., 0|\mathrm{H}, 6,1.07168457,4,120.94096494,2,180 ., 0| \mathrm{C}, 6,1.44008091,4$, $120.63967873,2,0 ., 0|\mathrm{C}, 8,1.44010635,6,116.91368336,4,180 ., 0| \mathrm{H}, 9,1.07168$ $737,8,118.41382394,6,0 ., 0|\mathrm{C}, 9,1.36567668,8,120.64050532,6,180 ., 0| \mathrm{H}, 11$, $1.07137297,9,120.83082042,8,180 ., 0 \mid C, 11,1.39484326,9,119.06889036,8,0$. , $0|\mathrm{H}, 13,1.07340343,11,119.47733871,9,180 ., 0| \mathrm{C}, 13,1.40767678,11,122.626$ $71471,9,0 ., 0|\mathrm{~N}, 15,1.34564983,13,120.109068,11,180 ., 0| \mathrm{H}, 16,1.01083971,1$ $5,111.41421114,13,0 ., 0|C, 15,1.46703878,13,118.26508055,11,0 ., 0| N, 1,1.3$ $4560638,18,121.63210147,15,0 ., 0 \mid \mathrm{H}, 19,1.01084124,1,111.41902019,18,180$. , 0||Version=x86-Win32-G98RevA.11.4| State=1-A' $|\mathrm{HF}=-491.4677471| \mathrm{MP} 2=-492$ $.3570141|\mathrm{MP} 3=-492.4070023| \mathrm{MP} 4 \mathrm{D}=-492.4328438|\mathrm{MP} 4 \mathrm{DQ}=-492.4203767| \mathrm{PUHF}=-4$ $91.8125209|\mathrm{PMP} 2-0=-492.6984196| \mathrm{PMP} 3-0=-492.743202|\mathrm{MP} 4 \mathrm{SDQ}=-492.436582| \mathrm{C}$ $\mathrm{CSD}=-492.4620439|\mathrm{CCSD}(\mathrm{T})=-492.501341| \mathrm{S} 2=1.623602|\mathrm{~S} 2-1=1.431873| \mathrm{S} 2 \mathrm{~A}=4.7$ $14562|\mathrm{RMSD}=3.239 \mathrm{e}-009| \mathrm{PG}=\mathrm{CS} \quad[\mathrm{SG}(\mathrm{C} 10 \mathrm{H} 8 \mathrm{~N} 2)]|| \mathrm{e}$

\section{UB3LYP/4-31G}

1 | 1 | UNPC-UNK | SP | UB3LYP | 4-31G | C10H8N2 | PCUSER | 31-Jan-2003|0|| \#UB3LYP / 4-3 $1 \mathrm{G}$ GUESS $=($ ALWAYS, MIX) || molecule 54|| $0,1|\mathrm{C}| \mathrm{C}, 1,1.4077918 \mid \mathrm{H}, 2,1.07340123$ , 1, 117.88745411|C, 2, 1.39472344,1,122.62485351,3,180.,0|H,4,1.07137734, $2,120.11043227,1,180 ., 0|\mathrm{C}, 4,1.36576681,2,119.07131078,1,0 ., 0| \mathrm{H}, 6,1.071$ $68457,4,120.94096494,2,180 ., 0|C, 6,1.44008091,4,120.63967873,2,0 ., 0| C, 8$ $, 1.44010635,6,116.91368336,4,180 ., 0 \mid \mathrm{H}, 9,1.07168737,8,118.41382394,6,0$. 
$, 0|\mathrm{C}, 9,1.36567668,8,120.64050532,6,180 ., 0| \mathrm{H}, 11,1.07137297,9,120.830820$ $42,8,180 ., 0|\mathrm{C}, 11,1.39484326,9,119.06889036,8,0 ., 0| \mathrm{H}, 13,1.07340343,11,1$ $19.47733871,9,180 ., 0|C, 13,1.40767678,11,122.62671471,9,0 ., 0| N, 15,1.345$ $64983,13,120.109068,11,180 ., 0|\mathrm{H}, 16,1.01083971,15,111.41421114,13,0 ., 0|$ $\mathrm{C}, 15,1.46703878,13,118.26508055,11,0 ., 0 \mid \mathrm{N}, 1,1.34560638,18,121.63210147$ $, 15,0 ., 0|\mathrm{H}, 19,1.01084124,1,111.41902019,18,180 ., 0| \mid$ Version=x86-Win32-G 98RevA. $11.4 \mid$ State=1-A' $|\mathrm{HF}=-494.6256925| \mathrm{S} 2=1.004645|\mathrm{~S} 2-1=0.| \mathrm{S} 2 \mathrm{~A}=0.37740$ $5|\mathrm{RMSD}=3.781 \mathrm{e}-005| \mathrm{Dipole}=-1.3323955,0 ., 0.8944949|\mathrm{PG}=\mathrm{CS} \quad[\mathrm{SG}(\mathrm{C} 10 \mathrm{H} 8 \mathrm{~N} 2)]| \mid$ a

\section{UHF/6-311G(d)}

1 | 1 | UNPC-UNK |FOpt | UHF | 6-311G (d) |C10H8N2 | PCUSER | 27-Nov-2003|0|| \# T UHF/6 -311G (D) GUESS=READ OPT SCF (CONVER=5) GEOM=CHECKPOINT $\mid$ mol5-uhf-6-311g d-opt-1||0,1|C,-0.8513824102,-0.5074641147,-1.280032384|C, -0.810132009 $8,-1.978322063,-1.2180134278 \mid \mathrm{H},-1.3316299297,-2.500934225,-2.002072644$ $6|\mathrm{C},-0.1752243283,-2.7009507209,-0.2634454413| \mathrm{H},-0.1880811174,-3.77492$ $52196,-0.2827753055|\mathrm{C}, 0.5004513425,-2.0120622474,0.7524162085| \mathrm{H}, 1.0140$ $928002,-2.5532828643,1.5246634286 \mid C, 0.509323395,-0.6117366919,0.765755$ $1198|\mathrm{C}, 1.2211184304,-0.0556798949,1.8359213403| \mathrm{H}, 1.696104114,-0.701840$ $11,2.5500505608|\mathrm{C}, 1.3118204251,1.3358429311,1.9722895447| \mathrm{H}, 1.855411529$ $4,1.772339182,2.7895653173|\mathrm{C}, 0.6973245465,2.1138443174,1.0484101984| \mathrm{H}$, $0.7626664931,3.1843160766,1.1466501981 \mid C,-0.0698863879,1.6139695462,-0$ $.1050724547|\mathrm{~N},-0.6093434674,2.441855722,-0.9161328233| \mathrm{H},-0.4203501765$, $3.3848248739,-0.6319860877|C,-0.1420997059,0.1706612244,-0.2136433911|$ $\mathrm{N},-1.4827636899,0.0708917083,-2.2292985128 \mid \mathrm{H},-1.8913433971,-0.60834344$ $27,-2.8435879913||$ Version=x86-Win32-G98RevA.11.4 |State=1-A' $\mid \mathrm{HF}=-492.18$ $14715|\mathrm{~S} 2=0.| \mathrm{S} 2-1=0 .|\mathrm{S} 2 \mathrm{~A}=0.| \mathrm{RMSD}=2.716 \mathrm{e}-006|\mathrm{RMSF}=4.487 \mathrm{e}-005| \mathrm{Dipole}=-2.4$ $653691,0 ., 1.6396426|\mathrm{PG}=\mathrm{CS} \quad[\mathrm{SG}(\mathrm{C} 10 \mathrm{H} 8 \mathrm{~N} 2)]| \mid \mathrm{Q}$

\section{UB3LYP/6-311G(d)}

1 | 1 | UNPC-UNK | SP | UB3LYP |6-311G (d) |C10H8N2 | PCUSER | 28-NOV-2003|0|| \#P UB3L YP /6-311G (D) GUESS=READ SP SCF (CONVER=5) GEOM=CHECKPOINT||mol5-ub31yp6-311gd-sp-1||0,1|C, $-0.8513824102,-0.5074641147,-1.280032384 \mid \mathrm{C},-0.8101$ $320098,-1.978322063,-1.2180134278 \mid \mathrm{H},-1.3316299297,-2.500934225,-2.0020$ $726446|\mathrm{C},-0.1752243283,-2.7009507209,-0.2634454413| \mathrm{H},-0.1880811174,-3$. $7749252196,-0.2827753055|\mathrm{C}, 0.5004513425,-2.0120622474,0.7524162085| \mathrm{H}, 1$ $.0140928002,-2.5532828643,1.5246634286 \mid C, 0.509323395,-0.6117366919,0.7$ $657551198|\mathrm{C}, 1.2211184304,-0.0556798949,1.8359213403| \mathrm{H}, 1.696104114,-0.7$ $0184011,2.5500505608|\mathrm{C}, 1.3118204251,1.3358429311,1.9722895447| \mathrm{H}, 1.8554$ $115294,1.772339182,2.7895653173 \mid \mathrm{C}, 0.6973245465,2.1138443174,1.04841019$ $84|\mathrm{H}, 0.7626664931,3.1843160766,1.1466501981| \mathrm{C},-0.0698863879,1.61396954$ $62,-0.1050724547|\mathrm{~N},-0.6093434674,2.441855722,-0.9161328233| \mathrm{H},-0.420350$ $1765,3.3848248739,-0.6319860877 \mid C,-0.1420997059,0.1706612244,-0.213643$ $3911|\mathrm{~N},-1.4827636899,0.0708917083,-2.2292985128| \mathrm{H},-1.8913433971,-0.608$ $3434427,-2.8435879913 \mid$ |Version=x86-Win32-G98RevA.11.4 | State=1-A' | HF=-4 $95.3705866|\mathrm{~S} 2=0.| \mathrm{S} 2-1=0 .|\mathrm{S} 2 \mathrm{~A}=0.| \mathrm{RMSD}=3.200 \mathrm{e}-006 \mid \mathrm{Dipole}=1.0942748,-1.31$ $46348,1.6452151|\mathrm{PG}=\mathrm{CS} \quad[\mathrm{SG}(\mathrm{C} 10 \mathrm{H} 8 \mathrm{~N} 2)]| \mid \mathrm{e}$

\section{RHF/STO-3G}


1 | 1 | UNPC-UNK |FOpt |ROHF |STO-3G |C10H8N2 | PCUSER | 27-NOV-2003|0|| \#ROHF/STO3G SCF (CONVER=5) OPT | molecule 54||0,1|C,1.5246734819,0.,0.5107057703| $\mathrm{C}, 1.479136834,0 ., 1.979230468|\mathrm{H}, 2.4347270207,0 ., 2.5010064148| \mathrm{C}, 0.326099$ $6823,0 ., 2.7125045746|\mathrm{H}, 0.3579249733,0 ., 3.7933110713| \mathrm{C},-0.9017942811,0$. ,2.0448875153|H, -1.8308275763,0.,2.6078404392|C, $-0.9431704013,0 ., 0.627$ $3745369|\mathrm{C},-2.2339888868,0 ., 0.0415085291| \mathrm{H},-3.1124567388,0 ., 0.680524375$ $|\mathrm{C},-2.3753620417,0 .,-1.3495644905| \mathrm{H},-3.3600680795,0 .,-1.7961963601 \mid \mathrm{C},-$ $1.253775444,0 .,-2.1295294874|\mathrm{H},-1.3653150875,0 .,-3.2125115416| \mathrm{C}, 0.1182$ $423894,0 .,-1.6034738305|\mathrm{~N}, 1.1673958748,0 .,-2.4039897458| \mathrm{H}, 0.7935210951$ $, 0 .,-3.3849549742|\mathrm{C}, 0.2271019297,0 .,-0.1510181025| \mathrm{N}, 2.6684549378,0 .,-0$ $.1476141727|\mathrm{H}, 3.4285591308,0 ., 0.5764551054| \mid$ Version=x86-Win32-G98RevA. $11.4 \mid$ State=1-A' $|H F=-485.9444296| \mathrm{RMSD}=3.829 \mathrm{e}-006|\mathrm{RMSF}=6.768 \mathrm{e}-005| \mathrm{Dipole}$ $=-2.265695,0 ., 1.5052748|\mathrm{PG}=\mathrm{CS} \quad[\mathrm{SG}(\mathrm{C} 10 \mathrm{H} 8 \mathrm{~N} 2)]| \mid \mathrm{e}$

\section{RHF/6-311G(d)}

1 | 1 | UNPC-UNK |FOpt |ROHF | 6-311G (d) |C10H8N2 | PCUSER | 01-DeC-2003 | 0 | | \#ROHF /6 -311G (D) SCF (CONVER=5) OPT GUESS=READ GEOM=CHECKPOINT ||molecule 54||0, $1|\mathrm{C}, 1.537314188,0 ., 0.5074641147| \mathrm{C}, 1.4628296495,0 ., 1.978322063 \mid \mathrm{H}, 2.4044$ $818868,0 ., 2.500934225|\mathrm{C}, 0.3163970066,0 ., 2.7009507209| \mathrm{H}, 0.3396121025,0$. , 3.7749252196|C, $-0.9036491006,0 ., 2.0120622474 \mid \mathrm{H},-1.8311151733,0 ., 2.553$ $2828643|\mathrm{C},-0.919669084,0 ., 0.6117366919| \mathrm{C},-2.2049347812,0 \ldots, 0.0556798949$ $|\mathrm{H},-3.0626013499,0 ., 0.70184011| \mathrm{C},-2.3687124933,0 .,-1.3358429311 \mid \mathrm{H},-3.3$ $50257692,0 .,-1.772339182|\mathrm{C},-1.2591367945,0 .,-2.1138443174| \mathrm{H},-1.3771226$ $73,0 .,-3.1843160766|\mathrm{C}, 0.1261916319,0 .,-1.6139695462| \mathrm{N}, 1.1002721532,0 .$, $-2.441855722|\mathrm{H}, 0.7590129683,0 .,-3.3848248739| \mathrm{C}, 0.2565849274,0 .,-0.1706$ $612244|\mathrm{~N}, 2.6773793192,0 .,-0.0708917083| \mathrm{H}, 3.4151387249,0 ., 0.6083434427 \mid$ |Version=x86-Win32-G98RevA.11.4| State=1-A ' $|\mathrm{HF}=-492.1814713| \mathrm{RMSD}=3.405 \mathrm{e}$ $-006|\mathrm{RMSF}=4.654 \mathrm{e}-005| \mathrm{Dipole}=-2.467068,0 ., 1.6407528 \mid \mathrm{PG}=\mathrm{CS} \quad[\mathrm{SG}(\mathrm{C} 10 \mathrm{H} 8 \mathrm{~N} 2)]$ || e

\section{RHF/6-311G(d,p)}

1 | 1 |UNPC-UNK |FOpt |ROHF |6-311G (d,p) |C10H8N2 | PCUSER | 14-DeC-2003|0||\#P RO $\mathrm{HF} / 6-311 * *$ OPT SCF (CONVER=5) GEOM=CHECKPOINT GUESS=READ $\mid$ molecule 5 $\mid$ $0,1|\mathrm{C}, 1.5374837457,0 ., 0.5075660326| \mathrm{C}, 1.4628625556,0 ., 1.9784810423 \mid \mathrm{H}, 2$. $4059633444,0 ., 2.4992553704|\mathrm{C}, 0.3165662382,0 ., 2.7010379| \mathrm{H}, 0.3395067982$, $0 ., 3.7750739181|\mathrm{C},-0.9035270184,0 ., 2.0121373754| \mathrm{H},-1.8323592133,0 ., 2.5$ $517675738|C,-0.9197157897,0 ., 0.6117647828| C,-2.204974782,0 ., 0.05553668$ $17|\mathrm{H},-3.0616925246,0 ., 0.703584148| \mathrm{C},-2.3687296853,0 .,-1.3360184106 \mid \mathrm{H},-$ $3.3504238611,0 .,-1.7723166614|\mathrm{C},-1.2592580552,0 .,-2.1139231092| \mathrm{H},-1.37$ $5005248,0 .,-3.1850197219|\mathrm{C}, 0.1261730234,0 .,-1.6141705851| \mathrm{N}, 1.100133145$ $3,0 .,-2.4419505569|\mathrm{H}, 0.7563583391,0 .,-3.3853202102| \mathrm{C}, 0.2565174171,0 .,-$ $0.1706223678|\mathrm{~N}, 2.677396609,0 .,-0.0707262258| \mathrm{H}, 3.414558189,0 ., 0.6109770$ $103 \mid$ Version=x86-Win32-G98RevA.11.4 | State=1-A' $|\mathrm{HF}=-492.2000386| \mathrm{RMSD}=2$. $272 \mathrm{e}-006|\mathrm{RMSF}=4.688 \mathrm{e}-005| \mathrm{Dipole}=-2.4520843,0 ., 1.6309313 \mid \mathrm{PG}=\mathrm{CS} \quad[\mathrm{SG}(\mathrm{C} 10 \mathrm{H}$ $8 \mathrm{~N} 2)]|| @$

\section{RB3LYP/6-311G(d,p)}

1 | 1 | UNPC-UNK | FOpt |ROB3LYP | 6-311G (d,p) |C10H8N2 | PCUSER | 14-Dec-2003|0|| \#P ROB3LYP/6-311** OPT SCF (CONVER=5) GEOM=CHECKPOINT GUESS=READ||molecu le 5|| $0,1|C, 1.5294222196,0 ., 0.518637112| C, 1.4844198839,0 ., 1.9666326903$ 
$|\mathrm{H}, 2.4420668657,0 ., 2.4802590081| \mathrm{C}, 0.3216530195,0 ., 2.7115815908 \mid \mathrm{H}, 0.361$ $9316316,0 ., 3.7946013636|\mathrm{C},-0.8967649098,0 ., 2.0453604174| \mathrm{H},-1.829647540$ $3,0 ., 2.5971728421|C,-0.936538003,0 ., 0.6251908393| C,-2.2330219796,0 ., 0$. $0440035757|\mathrm{H},-3.1005133917,0 ., 0.6939817742| \mathrm{C},-2.3810842034,0 .,-1.33702$ $18476|\mathrm{H},-3.3660264613,0 .,-1.7896035832| \mathrm{C},-1.2470216638,0 .,-2.125417220$ $3|\mathrm{H},-1.354243398,0 .,-3.2070315611| \mathrm{C}, 0.1076972668,0 .,-1.6115626014 \mid \mathrm{N}, 1$. $1386317171,0 .,-2.4255764768|\mathrm{H}, 0.7913386406,0 .,-3.3889507239| \mathrm{C}, 0.237271$ $3292,0 .,-0.1584433966|\mathrm{~N}, 2.6765635428,0 .,-0.1207295861| \mathrm{H}, 3.4325290785,0$ ., 0.5699463637|| Version=x86-Win32-G98RevA. $11.4 \mid$ State $^{\prime}{ }^{\prime}-\mathrm{A}^{\prime} \mid \mathrm{HF}=-495.3938$ $619|\mathrm{RMSD}=5.943 \mathrm{e}-006| \mathrm{RMSF}=6.840 \mathrm{e}-005|\mathrm{Dipole}=-1.8767578,0 ., 1.2703186| \mathrm{PG}=$ CS [SG (C10H8N2)] || @

\section{Triplet}

\section{UHF/STO-3G}

$1|1|$ UNPC-UNK |FOpt | UHF |STO-3G |C10H8N2 (3) | PCUSER |19-OCt-2002|0||\#UHF STO -3G OPT GUESS $=($ ALWAYS, MIX) || molecule 54|| $0,3 \mid \mathrm{C}, 1.5056753646,0 ., 0.56070$ $81818|\mathrm{C}, 1.5120817801,0 ., 2.0028744501| \mathrm{H}, 2.469343317,0 ., 2.5105280047 \mid \mathrm{C}, 0$ $.3182453967,0 ., 2.752852264|\mathrm{H}, 0.3576644608,0 ., 3.8347249071| \mathrm{C},-0.9238224$ $766,0 ., 2.0865925609|\mathrm{H},-1.8456798651,0 ., 2.6540595721| \mathrm{C},-0.9962140596,0$. $, 0.6516451771|\mathrm{C},-2.2819309811,0 ., 0.010360108| \mathrm{H},-3.171226188,0 ., 0.62760$ $71334|\mathrm{C},-2.3946941956,0 .,-1.3946022053| \mathrm{H},-3.3701296252,0 .,-1.864206292$ $7|\mathrm{C},-1.2292022682,0 .,-2.1879120765| \mathrm{H},-1.3107648512,0 .,-3.2683792561 \mid \mathrm{C}$, $0.0895678347,0 .,-1.6041912694|\mathrm{~N}, 1.2312360526,0 .,-2.4259003664| \mathrm{H}, 0.8528$ $884167,0 .,-3.4110207771|\mathrm{C}, 0.220356712,0 .,-0.1441400756| \mathrm{N}, 2.7159858396$, $0 .,-0.156062825|\mathrm{H}, 3.4669724473,0 ., 0.5853063578| \mid$ Version=x86-Win32-G98R evA.11.4 State=3-A' $|\mathrm{HF}=-486.1937659| \mathrm{S} 2=3.837797|\mathrm{~S} 2-1=0.| \mathrm{S} 2 \mathrm{~A}=5.505987 \mid \mathrm{R}$ $\mathrm{MSD}=3.136 \mathrm{e}-009|\mathrm{RMSF}=1.806 \mathrm{e}-005| \mathrm{Dipole}=-0.8620821,0 ., 0.5639066 \mid \mathrm{PG}=\mathrm{CS} \quad[\mathrm{S}$ $\mathrm{G}(\mathrm{C} 10 \mathrm{H} 8 \mathrm{~N} 2)]||$ e

\section{UCCSD(T)/STO-3G}

1 | 1 | UNPC-UNK | SP | UCCSD (T) -FC $\mid$ STO-3G | C10H8N2 (3) | PCUSER | 19-OCt-2002|0|| \#U CCSD-T/STO-3G GUESS = (ALWAYS, MIX) | molecule 54||0,3|C|C, 1, 1.4421805|H,2 , 1.08354132,1,118.19235567|C,2,1.40986242,1,121.88285686,3,180.,0|H,4, $1.08259054,2,120.05067206,1,180 ., 0 \mid C, 4,1.40948026,2,119.6529964,1,0 ., 0$ $|\mathrm{H}, 6,1.08251552,4,120.17519948,2,180 ., 0| \mathrm{C}, 6,1.43677226,4,121.09769134$, $2,0 ., 0|\mathrm{C}, 8,1.43677227,6,119.39699452,4,180 ., 0| \mathrm{H}, 9,1.08251552,8,118.727$ $10939,6,0 ., 0|C, 9,1.40948027,8,121.09769249,6,180 ., 0| \mathrm{H}, 11,1.08259054,9$, $120.2963303,8,180 ., 0|\mathrm{C}, 11,1.4098624,9,119.65299748,8,0 ., 0| \mathrm{H}, 13,1.08354$

$131,11,119.92478782,9,180 ., 0|C, 13,1.44218049,11,121.8828554,9,0 ., 0| \mathrm{N}, 1$ $5,1.40663149,13,120.38037979,11,180 ., 0 \mid \mathrm{H}, 16,1.05527682,15,104.73433281$ $, 13,0 ., 0|\mathrm{C}, 15,1.46589741,13,118.99418108,11,0 ., 0| \mathrm{N}, 1,1.40663148,18,120$ $.62544005,15,0 ., 0|\mathrm{H}, 19,1.05527681,1,104.73433661,18,180 ., 0| \mid$ Version $=x 8$ 6-Win32-G98RevA. $11.4 \mid$ State $=3-\mathrm{B} 2|\mathrm{HF}=-486.1937659| \mathrm{MP} 2=-486.6911717 \mid \mathrm{MP} 3=-$ $486.7847035|\mathrm{MP} 4 \mathrm{D}=-486.814577| \mathrm{MP} 4 \mathrm{DQ}=-486.8096298|\mathrm{PUHF}=-486.3349238| \mathrm{PMP} 2$ $-0=-486.8260005|\mathrm{PMP} 3-0=-486.9099417| \mathrm{MP} 4 \mathrm{SDQ}=-486.8231572 \mid \mathrm{CCSD}=-486.8913$ $36|\operatorname{CCSD}(\mathrm{T})=-486.9089438| \mathrm{S} 2=3.837797|\mathrm{~S} 2-1=3.45355| \mathrm{S} 2 \mathrm{~A}=5.505987 \mid \mathrm{RMSD}=3.2$ $10 \mathrm{e}-009|\mathrm{PG}=\mathrm{C} 02 \mathrm{~V} \quad[\mathrm{C} 2(\mathrm{C} 1 \mathrm{C} 1), \mathrm{SGV}(\mathrm{C} 8 \mathrm{H} 8 \mathrm{~N} 2)]| \mid \mathrm{e}$ 


\section{UB3LYP/STO-3G}

1 | 1 | UNPC-UNK | SP | UB3LYP |STO-3G|C10H8N2 (3) |PCUSER $02-F e b-2003|0| \mid$ \#UB3LYP /STO-3G GUESS=(ALWAYS, MIX) || molecule 54||0,3|C|C,1,1.4421805| H, 2, 1.083 $54132,1,118.19235567|\mathrm{C}, 2,1.40986242,1,121.88285686,3,180 ., 0| \mathrm{H}, 4,1.0825$ $9054,2,120.05067206,1,180 ., 0|\mathrm{C}, 4,1.40948026,2,119.6529964,1,0 ., 0| \mathrm{H}, 6,1$ $.08251552,4,120.17519948,2,180 ., 0 \mid C, 6,1.43677226,4,121.09769134,2,0 ., 0$ $|\mathrm{C}, 8,1.43677227,6,119.39699452,4,180 ., 0| \mathrm{H}, 9,1.08251552,8,118.72710939$, $6,0 ., 0|\mathrm{C}, 9,1.40948027,8,121.09769249,6,180 ., 0| \mathrm{H}, 11,1.08259054,9,120.29$ $63303,8,180 ., 0|\mathrm{C}, 11,1.4098624,9,119.65299748,8,0 ., 0| \mathrm{H}, 13,1.08354131,11$ $, 119.92478782,9,180 ., 0|C, 13,1.44218049,11,121.8828554,9,0 ., 0| N, 15,1.40$ $663149,13,120.38037979,11,180 ., 0 \mid \mathrm{H}, 16,1.05527682,15,104.73433281,13,0$. , $0|\mathrm{C}, 15,1.46589741,13,118.99418108,11,0 ., 0| \mathrm{N}, 1,1.40663148,18,120.62544$ $005,15,0 ., 0|\mathrm{H}, 19,1.05527681,1,104.73433661,18,180.0| \mid$ Version=x86-Win3 2-G98RevA. 11.4 $|\mathrm{HF}=-489.1137043| \mathrm{S} 2=2.161442|\mathrm{~S} 2-1=0.| \mathrm{S} 2 \mathrm{~A}=2.014495 \mid \mathrm{RMSD}=1$ $.708 e-004 \mid$ Dipole $=-0.9400433,-0.0009514,0.6089573 \mid \mathrm{PG}=\mathrm{C} 02 \mathrm{~V}$ [C2 (C1C1), SGV $(\mathrm{C} 8 \mathrm{H} 8 \mathrm{~N} 2)]|| \mathrm{e}$

\section{UHF/4-31G}

1 | 1 |UNPC-UNK |FOpt | UHF | 4-31G |C10H8N2 (3) |PCUSER |05-Dec-2002|0|| \#UHF 4-31 G OPT GUESS $=$ (ALWAYS, MIX) || molecule 54||0,3|C,1.5018584536,0.,0.5639037 $395|\mathrm{C}, 1.4811338568,0 ., 1.9920553999| \mathrm{H}, 2.4248992424,0 ., 2.5035737083 \mid \mathrm{C}, 0$. $3000046061,0 ., 2.7207418449|\mathrm{H}, 0.3259913163,0 ., 3.7920011621| \mathrm{C},-0.9154208$ $601,0 ., 2.046189689|\mathrm{H},-1.837182468,0 ., 2.5940736277| \mathrm{C},-0.9693552229,0 ., 0$ $.6279454421|\mathrm{C},-2.2416258524,0 .,-0.0010641799| \mathrm{H},-3.1184586755,0 ., 0.6161$ $760264|\mathrm{C},-2.3603619777,0 .,-1.3860484424| \mathrm{H},-3.3273920943,0 .,-1.84770262$ $83|\mathrm{C},-1.2124937304,0 .,-2.1660796781| \mathrm{H},-1.2934982637,0 .,-3.236491911 \mid \mathrm{C}$, $0.0993397078,0 .,-1.6011551657|\mathrm{~N}, 1.1704197979,0 .,-2.4102854418| \mathrm{H}, 0.9007$ $214332,0 .,-3.3843408873|\mathrm{C}, 0.2382662735,0 .,-0.1543481862| \mathrm{N}, 2.678152571$, $0 .,-0.0828083392|\mathrm{H}, 3.4568414017,0 ., 0.56152459| \mid$ Version $=\mathrm{x} 86-\mathrm{Win} 32-\mathrm{G} 98 \mathrm{Re}$ $\mathrm{VA} .11 .4 \mid$ State=3-B2 $|\mathrm{HF}=-491.5060676| \mathrm{S} 2=3.233991|\mathrm{~S} 2-1=0.| \mathrm{S} 2 \mathrm{~A}=3.406274 \mid \mathrm{RM}$ $\mathrm{SD}=3.262 \mathrm{e}-009|\mathrm{RMSF}=3.877 \mathrm{e}-005| \mathrm{Dipole}=-1.0799689,0 ., 0.6996007 \mid \mathrm{PG}=\mathrm{C} 02 \mathrm{~V} \quad[$ $\mathrm{C} 2(\mathrm{C} 1 \mathrm{C} 1), \mathrm{SGV}(\mathrm{C} 8 \mathrm{H} 8 \mathrm{~N} 2)]$ || e

\section{UCCSD(T)/4-31G}

1 | 1 | UNPC-UNK | SP | UCCSD ( T) -RW | 4-31G |C10H8N2 (3) |PCUSER | 26-Mar-2003|0|| \#UC $\operatorname{CSD}(\mathrm{T}, \mathrm{WINDOW}=(13,110)) / 4-31 \mathrm{G}$ GUESS=(ALWAYS,MIX) || molecule 54|| $0,3|\mathrm{C}| \mathrm{C}$, $1,1.42830202|\mathrm{H}, 2,1.07347291,1,117.62618566| \mathrm{C}, 2,1.38782212,1,122.503504$ $03,3,180 ., 0|\mathrm{H}, 4,1.07157446,2,120.2825018,1,180 ., 0| \mathrm{C}, 4,1.39006463,2,119$ $.29793316,1,0 ., 0|\mathrm{H}, 6,1.07229719,4,120.24331175,2,180 ., 0| \mathrm{C}, 6,1.41926941$ $, 4,121.20780093,2,0 ., 0|\mathrm{C}, 8,1.41926941,6,118.48560599,4,180 ., 0| \mathrm{H}, 9,1.07$ $229719,8,118.54888732,6,0 ., 0|\mathrm{C}, 9,1.39006463,8,121.20780093,6,180 ., 0| \mathrm{H}$, $11,1.07157446,9,120.41956505,8,180 ., 0 \mid \mathrm{C}, 11,1.38782212,9,119.29793316,8$ $, 0 ., 0|\mathrm{H}, 13,1.07347291,11,119.87031031,9,180 ., 0| \mathrm{C}, 13,1.42830202,11,122$. $50350403,9,0 ., 0|\mathrm{~N}, 15,1.34235031,13,119.63284388,11,180 ., 0| \mathrm{H}, 16,1.01070$ $333,15,111.59218882,13,0 ., 0|\mathrm{C}, 15,1.45346174,13,118.78340718,11,0 ., 0| \mathrm{N}$, $1,1.34235031,18,121.58374894,15,0 ., 0 \mid \mathrm{H}, 19,1.01070333,1,111.59218882,18$ ,180.,0||Version=x86-Win32-G98RevA.11.4| State=3-B2 | HF=-491.5060676|MP2 $=-492.3418518|\mathrm{MP} 3=-492.4013261| \mathrm{MP} 4 \mathrm{D}=-492.4246062|\mathrm{MP} 4 \mathrm{DQ}=-492.4154546| \mathrm{PU}$ $\mathrm{HF}=-491.5831408|\mathrm{PMP} 2-0=-492.4132796| \mathrm{PMP} 3-0=-492.463841 \mid \mathrm{MP} 4 \mathrm{SDQ}=-492.433$ 
$5284|\mathrm{CCSD}=-492.4704206| \operatorname{CCSD}(\mathrm{T})=-492.5068735|\mathrm{~S} 2=3.23399| \mathrm{S} 2-1=2.929566 \mid \mathrm{S}$ $2 \mathrm{~A}=3.406271|\mathrm{RMSD}=5.863 \mathrm{e}-009| \mathrm{PG}=\mathrm{C} 02 \mathrm{~V} \quad[\mathrm{C} 2(\mathrm{C} 1 \mathrm{C} 1), \mathrm{SGV}(\mathrm{C} 8 \mathrm{H} 8 \mathrm{~N} 2)]|| \mathrm{e}$

\section{UB3LYP/4-31G}

1 | 1 | UNPC-UNK | SP | UB3LYP | 4-31G |C10H8N2 (3) |PCUSER | 31-Jan-2003|0|| \#UB3LYP / 4-31G GUESS = (ALWAYS, MIX) || molecule 54||0,3|C|C,1,1.42830202| H, 2, 1.0734 $7291,1,117.62618566|\mathrm{C}, 2,1.38782212,1,122.50350403,3,180 ., 0| \mathrm{H}, 4,1.07157$ $446,2,120.2825018,1,180 ., 0|\mathrm{C}, 4,1.39006463,2,119.29793316,1,0 ., 0| \mathrm{H}, 6,1$. $07229719,4,120.24331175,2,180 ., 0|C, 6,1.41926941,4,121.20780093,2,0 ., 0|$ $\mathrm{C}, 8,1.41926941,6,118.48560599,4,180 ., 0 \mid \mathrm{H}, 9,1.07229719,8,118.54888732,6$ $, 0 ., 0|\mathrm{C}, 9,1.39006463,8,121.20780093,6,180 ., 0| \mathrm{H}, 11,1.07157446,9,120.419$ $56505,8,180 ., 0|\mathrm{C}, 11,1.38782212,9,119.29793316,8,0.0| \mathrm{H}, 13,1.07347291,1$ $1,119.87031031,9,180 ., 0|C, 13,1.42830202,11,122.50350403,9,0 ., 0| N, 15,1$. $34235031,13,119.63284388,11,180 ., 0 \mid \mathrm{H}, 16,1.01070333,15,111.59218882,13$, $0 ., 0|C, 15,1.45346174,13,118.78340718,11,0 ., 0| N, 1,1.34235031,18,121.583$ $74894,15,0.0|\mathrm{H}, 19,1.01070333,1,111.59218882,18,180,0| \mid$ Version=x86-Wi n32-G98RevA. 11.4 $|\mathrm{HF}=-494.6326772| \mathrm{S} 2=2.104963|\mathrm{~S} 2-1=0.| \mathrm{S} 2 \mathrm{~A}=2.00616 \mid \mathrm{RMSD}=$ $5.281 \mathrm{e}-005|\mathrm{Dipole}=-1.1982194,0.0034428,0.8011218| \mathrm{PG}=\mathrm{C} 02 \mathrm{~V}$ [C2 (C1C1), SGV $(\mathrm{C} 8 \mathrm{H} 8 \mathrm{~N} 2)]||$ e

\section{UHF/6-311G(d)}

1 | 1 | UNPC-UNK |FOpt | UHF | 6-311G (d) |C10H8N2 (3) |PCUSER | 29-NOV-2003|0||\#P UH F/6-311G (D) GUESS=READ OPT SCF (CONVER=5) GEOM = CHECKPOINT || mol5-uhf-6 $311 \mathrm{gd}-\mathrm{opt}-3|| 0,3|\mathrm{C}, 1.5115819537,0 ., 0.5626554046| \mathrm{C}, 1.4864510762,0 ., 1.99$ $72328357|\mathrm{H}, 2.4309328612,0 ., 2.5128337109| \mathrm{C}, 0.3032234837,0 ., 2.723656432 \mid$ $\mathrm{H}, 0.3280015472,0 ., 3.7982690507|\mathrm{C},-0.9138333239,0 ., 2.0475727458| \mathrm{H},-1.83$ $69849866,0 ., 2.5982728431|C,-0.9677028673,0 ., 0.6268750511| C,-2.24223906$ $33,0 .,-0.0030784018|\mathrm{H},-3.1222102357,0 ., 0.6142791327| \mathrm{C},-2.3617059915,0$. $,-1.3901775606|\mathrm{H},-3.3322905044,0 .,-1.852099563| \mathrm{C},-1.2150450507,0 .,-2.1$ $730488551|\mathrm{H},-1.299482551,0 .,-3.2457838705| \mathrm{C}, 0.1044539979,0 .,-1.6095187$ $074|\mathrm{~N}, 1.1674963513,0 .,-2.4080779904| \mathrm{H}, 0.8554864559,0 .,-3.3630487957 \mid \mathrm{C}$, $0.2382416808,0 .,-0.1543322552|\mathrm{~N}, 2.6749428795,0 .,-0.081042762| \mathrm{H}, 3.41891$ $74239,0 ., 0.5941026232||$ Version=x86-Win32-G98RevA.11.4 | State=3-B2 $\mid \mathrm{HF}=-4$ $92.3102352|\mathrm{~S} 2=3.164372| \mathrm{S} 2-1=0 .|\mathrm{S} 2 \mathrm{~A}=3.228129| \mathrm{RMSD}=9.687 \mathrm{e}-007 \mid \mathrm{RMSF}=7.913$ e-005|Dipole $=-1.0123569,0 ., 0.6558018 \mid \mathrm{PG}=\mathrm{C} 02 \mathrm{~V} \quad$ [C2 (C1C1), SGV (C8H8N2) ] | | @

\section{UB3LYP/6-311G(d)}

1 | 1 | UNPC-UNK | SP | UB3LYP | 6-311G (d) |C10H8N2 (3) | PCUSER | 29-NOV-2003|0|| \#P U B3LYP /6-311G(D) GUESS=READ SP SCF (CONVER=5) GEOM = CHECKPOINT $\mid$ mol5-ub 31yp-6311gd-sp-3||0,3|C,1.5115819537,0.,0.5626554046|C, 1.4864510762, 0 . $, 1.9972328357|\mathrm{H}, 2.4309328612,0 ., 2.5128337109| \mathrm{C}, 0.3032234837,0 ., 2.72365$ $6432|\mathrm{H}, 0.3280015472,0 ., 3.7982690507| \mathrm{C},-0.9138333239,0 ., 2.0475727458 \mid \mathrm{H}$, $-1.8369849866,0 ., 2.5982728431|\mathrm{C},-0.9677028673,0 ., 0.6268750511| \mathrm{C},-2.242$ $2390633,0 .,-0.0030784018|\mathrm{H},-3.1222102357,0 ., 0.6142791327| \mathrm{C},-2.36170599$ $15,0 .,-1.3901775606|\mathrm{H},-3.3322905044,0 .,-1.852099563| \mathrm{C},-1.2150450507,0$. $,-2.1730488551|\mathrm{H},-1.299482551,0 .,-3.2457838705| \mathrm{C}, 0.1044539979,0 .,-1.60$ $95187074|\mathrm{~N}, 1.1674963513,0 .,-2.4080779904| \mathrm{H}, 0.8554864559,0 .,-3.36304879$ $57|\mathrm{C}, 0.2382416808,0 .,-0.1543322552| \mathrm{N}, 2.6749428795,0 .,-0.081042762 \mid \mathrm{H}, 3$. $4189174239,0 ., 0.5941026232||$ Version=x86-Win32-G98RevA.11.4|State=3-B2 | $\mathrm{HF}=-495.3981745|\mathrm{~S} 2=2.101924| \mathrm{S} 2-1=0 .|\mathrm{S} 2 \mathrm{~A}=2.005922| \mathrm{RMSD}=7.231 \mathrm{e}-006 \mid \mathrm{Dipol}$ 
$e=-1.182509,0 ., 0.7660258|P G=C 02 V \quad[C 2(C 1 C 1), S G V(C 8 H 8 N 2)]| \mid \varrho$

\section{ROHF/STO-3G}

1 | 1 | UNPC-UNK |FOpt |ROHF |STO-3G|C10H8N2 (3)|PCUSER |29-Nov-2003|0||\#P ROHF /STO-3G OPT ||mol5-rohf-sto3g-opt-1||0,3|C,1.4609795129,0.,0.5826505798 |C, 1.4710117102,0.,1.9474130799|H,2.4195484447,0.,2.4708288158|C,0.272 $772696,0 ., 2.7058385666|\mathrm{H}, 0.3284285769,0 ., 3.7867460248| \mathrm{C},-0.9182729197$, $0 ., 2.0678449983|\mathrm{H},-1.8469006258,0 ., 2.6241663177| \mathrm{C},-0.985416509,0 ., 0.63$ $83498957|\mathrm{C},-2.2625548822,0 .,-0.0073141699| \mathrm{H},-3.1498947437,0 ., 0.6127429$ $22|\mathrm{C},-2.3578935261,0 .,-1.355103469| \mathrm{H},-3.3215997771,0 .,-1.8477785871 \mid \mathrm{C}$, $-1.1758902183,0 .,-2.1385919082|\mathrm{H},-1.2658020327,0 .,-3.2182223241| \mathrm{C}, 0.06$ $55193572,0 .,-1.5715120264|\mathrm{~N}, 1.2616679299,0 .,-2.4208298026| \mathrm{H}, 0.86097063$ $43,0 .,-3.398937893|\mathrm{C}, 0.1987635137,0 .,-0.1287584154| \mathrm{N}, 2.7250784386,0$, , $0.1617726861|\mathrm{H}, 3.4539125347,0 ., 0.6037693568| \mid$ Version=x86-Win32-G98RevA $.11 .4 \mid$ State $=3-B 2|\mathrm{HF}=-486.0939073| \mathrm{RMSD}=8.536 \mathrm{e}-009|\mathrm{RMSF}=1.917 \mathrm{e}-005| \mathrm{Dipol}$ $\mathrm{e}=-0.8462242,0 ., 0.5481816|\mathrm{PG}=\mathrm{C} 02 \mathrm{~V} \quad[\mathrm{C} 2(\mathrm{C} 1 \mathrm{C} 1), \mathrm{SGV}(\mathrm{C} 8 \mathrm{H} 8 \mathrm{~N} 2)]| \mid \mathrm{e}$

\section{ROHF/6-311G(d)}

1 | 1 | UNPC-UNK |FOpt |ROHF |6-311G (d) |C10H8N2 (3) |PCUSER |29-NOV-2003|0||\#P R OHF $/ 6-311 \mathrm{G}$ (D) GUESS=READ OPT SCF (CONVER=5) GEOM = CHECKPOINT $\mid$ mol5-roh f-6311gd-opt-3||0,3|C, $1.4764368904,0$. $0.5736375614 \mid \mathrm{C}, 1.4653437926,0 ., 1$ $.9459895644|\mathrm{H}, 2.4064613798,0 ., 2.467776153| \mathrm{C}, 0.2754527329,0 ., 2.69407311$ $64|\mathrm{H}, 0.3176005066,0 ., 3.7679607316| \mathrm{C},-0.9107213207,0 ., 2.0430701949 \mid \mathrm{H},-1$ $.8357020391,0 ., 2.5912235607|\mathrm{C},-0.9637963376,0 ., 0.6243444127| \mathrm{C},-2.23685$ $77328,0 .,-0.0040778072|\mathrm{H},-3.1152524353,0 ., 0.615990083| \mathrm{C},-2.3460605126$, $0 .,-1.3527395298|\mathrm{H},-3.3088825151,0 .,-1.8302171067| \mathrm{C},-1.1769081719,0 .,-$ $2.1328373051|\mathrm{H},-1.2683661909,0 .,-3.2050308454| \mathrm{C}, 0.0800639085,0 .,-1.581$ $9341685|\mathrm{~N}, 1.1932604661,0 .,-2.4164243525| \mathrm{H}, 0.8591488315,0 .,-3.363962185$ $3|\mathrm{C}, 0.2235923435,0 .,-0.1448424579| \mathrm{N}, 2.6930924913,0,,-0.1011435819 \mid \mathrm{H}, 3$. $4212482067,0 ., 0.5911336625||$ Version=x86-Win32-G98RevA.11.4 | State=3-B2 | $\mathrm{HF}=-492.2395187|\mathrm{RMSD}=8.524 \mathrm{e}-006| \mathrm{RMSF}=1.482 \mathrm{e}-004 \mid \mathrm{Dipole}=-0.9353828,0 ., 0$ $.6059382|\mathrm{PG}=\mathrm{C} 02 \mathrm{~V} \quad[\mathrm{C} 2(\mathrm{C} 1 \mathrm{C} 1), \mathrm{SGV}(\mathrm{C} 8 \mathrm{H} 8 \mathrm{~N} 2)]| \mid \mathrm{e}$

\section{ROHF/6-311G(d,p)}

1 | 1 | UNPC-UNK |FOpt |ROHF |6-311G (d,p) |C10H8N2 (3) |PCUSER|15-Dec-2003|0||\#P ROHF / 6-311** OPT SCF (CONVER=5) GEOM=CHECKPOINT GUESS=READ||molecule 5|| $0,3|\mathrm{C}, 1.4766455555,0 ., 0.5737507318| \mathrm{C}, 1.4654024781,0 ., 1.9461558707 \mid \mathrm{H}$ ,2.4075324461,0.,2.4662397256|C,0.2756175539,0.,2.6938728875|H,0.31762 $20742,0 ., 3.7678442215|\mathrm{C},-0.9106849415,0 ., 2.043137048| \mathrm{H},-1.8361973748,0$ $.2 .5905598739|C,-0.9639938913,0 ., 0.6244723874| C,-2.2369038723,0 .,-0.0$ $041383376|\mathrm{H},-3.1148492375,0 ., 0.6167134565| \mathrm{C},-2.3458103996,0 .,-1.352808$ $094|\mathrm{H},-3.3087673686,0 .,-1.8301891595| \mathrm{C},-1.1770359555,0 .,-2.1329588499 \mid$ $\mathrm{H},-1.2665261558,0 .,-3.2053802208|\mathrm{C}, 0.0800459306,0 .,-1.5821708655| \mathrm{N}, 1.1$ $929904112,0 .,-2.4164097163|\mathrm{H}, 0.8570754243,0 .,-3.365142248| \mathrm{C}, 0.22353731$ $6,0 .,-0.1448068112|\mathrm{~N}, 2.6929687338,0 .,-0.1009031075| \mathrm{H}, 3.4214775335,0 \ldots$ .5935083151|| Version=x86-Win32-G98RevA.11.4| State=3-B2 |HF=-492.2576825 $|\mathrm{RMSD}=5.496 \mathrm{e}-006| \mathrm{RMSF}=3.159 \mathrm{e}-005|\mathrm{Dipole}=-0.9216655,0 ., 0.5970522| \mathrm{PG}=\mathrm{C} 02$ $\mathrm{V}[\mathrm{C} 2(\mathrm{C} 1 \mathrm{C} 1), \mathrm{SGV}(\mathrm{C} 8 \mathrm{H} 8 \mathrm{~N} 2)]||$ e 


\section{ROB3LYP/6-311G(d,p)}

1 | 1 | UNPC-UNK | FOpt |ROB3LYP | 6-311G (d,p) |C10H8N2 (3) |PCUSER | 15-Dec-2003|0 | \#P ROB3LYP/6-311** OPT SCF (CONVER=5) GEOM=CHECKPOINT GUESS=READ | mol ecule 5|| $0,3|C, 1.5175098954,0 ., 0.5627622589| C, 1.4794048682,0 ., 2.000115$ $0019|\mathrm{H}, 2.4334834533,0 ., 2.5172292312| \mathrm{C}, 0.300156597,0 ., 2.718323645 \mid \mathrm{H}, 0.3$ $206780058,0 ., 3.8023071882|\mathrm{C},-0.9096274546,0 ., 2.0437389864| \mathrm{H},-1.8435078$ $988,0 ., 2.5940276049|\mathrm{C},-0.962563824,0 \ldots, 0.623545994| \mathrm{C},-2.2370209059,0 \ldots,-$ $0.0053494988|\mathrm{H},-3.1210025538,0 ., 0.6219675613| \mathrm{C},-2.3580929429,0 .,-1.385$ $1985758|\mathrm{H},-3.3389697071,0 .,-1.8470667643| \mathrm{C},-1.2205559141,0 .,-2.1677965$ $877|\mathrm{H},-1.3024512952,0 .,-3.2499085131| \mathrm{C}, 0.1067798638,0 .,-1.6149723538 \mid \mathrm{N}$ $, 1.1695026231,0 .,-2.4010234417|\mathrm{H}, 0.8454642847,0 .,-3.373095254| \mathrm{C}, 0.2357$ $638087,0 .,-0.1527270969|\mathrm{~N}, 2.6693249286,0 .,-0.0857576756| \mathrm{H}, 3.4239888995$ , $0 ., 0.6073561275||$ Version=x86-Win32-G98RevA.11.4 State=3-B2 $\mid \mathrm{HF}=-495.40$ $77033|\mathrm{RMSD}=2.484 \mathrm{e}-006| \mathrm{RMSF}=8.357 \mathrm{e}-005|\mathrm{Dipole}=-1.2103546,0 ., 0.7840641| \mathrm{P}$ $\mathrm{G}=\mathrm{C} 02 \mathrm{~V}[\mathrm{C} 2(\mathrm{C} 1 \mathrm{C} 1), \mathrm{SGV}(\mathrm{C} 8 \mathrm{H} 8 \mathrm{~N} 2)]||$ e 